\title{
Correction to: Prevalence, socio-economic predictors and health correlates of food insecurity among Italian children- findings from a cross-sectional study
}

\author{
Drieda Zaçe $^{1}$ (D) Maria Luisa Di Pietro ${ }^{1} \cdot$ Laura Reali $^{2} \cdot$ Chiara de Waure $^{3} \cdot$ Walter Ricciardi $^{1,4}$ \\ Published online: 19 November 2020 \\ (C) International Society for Plant Pathology and Springer Nature B.V. 2020
}

\section{Correction to: Food Security} https://doi.org/10.1007/s12571-020-01111-1

The original version of this article unfortunately contained mistakes introduced during the production process. The following Abstract and Keywords were missing in the paper:

\footnotetext{
Abstract

Considering food insecurity is a problem, even in developed countries, and the evidence gap regarding this issue in Italy, we aimed to assess the prevalence of food insecurity in a sample of Italian children and examine socio-economic and health correlates. This cross-sectional study was conducted in paediatric practices. Parents answered to the 18-items of the Household Food Security Module, 8 of which concern children. Paediatricians answered a questionnaire on children's health. Socio-economic and health correlates of food insecurity in children were assessed using univariable and multivariable logistic regression. Among 573 households with children, 15.4\% were food insecure, while $9 \cdot 1 \%$ of children were food insecure. Socio-

Drieda Zaçe and Maria Luisa Di Pietro contributed equally to this work. The online version of the original article can be found at https://doi.org/ 10.1007/s12571-020-01111-1

Drieda Zaçe

drieda.zace@unicatt.it

1 Sezione di Igiene, Dipartimento Universitario di Scienze della Vita e Sanità Pubblica, Università Cattolica del Sacro Cuore, Largo

Francesco Vito, 1, Rome, Italy

2 Primary Care Paediatrician, ASL Roma 1, Rome, Italy

3 Department of Experimental Medicine, Università degli Studi di Perugia, Perugia, Italy

4 Department of Woman and Child Health and Public Health - Public Health Area, Fondazione Policlinico Universitario A. Gemelli IRCCS, Rome, Italy
}

economic factors associated to food insecurity were living in south Italy, households with three or more children, lower household yearly income, worse economic situation description and younger parents. Food insecure children were less likely to have a normal relational [aOR 0.31 (CI 0.11-0.85)] and physical development [aOR 0.32(CI 0.15-0.65) and had more school difficulties [aOR 3.1(CI 1.33-7.24)] compared to their food secure peers. Food insecure children had higher odds of a deterioration in their health since birth and of a worse perceived health status, as reported by their parents. Considering the results in this sample and the lack of research regarding this issues in Italy and Europe more broadly, we call for consistent, national monitoring to determine the magnitude of the problem of food insecurity in households with children in Italy and to examine the socioeconomic variables and health implications in different contexts.

Keywords Food security/insecurity · Households · Children · Health · Italy

The original article has been corrected. 